\title{
Pseudomonas syringae Effector AvrPphB Suppresses AvrB-Induced Activation of RPM1 but Not AvrRpm1-Induced Activation
}

\author{
Andrew R. Russell, Tom Ashfield, and Roger W. Innes \\ Department of Biology, Indiana University, Bloomington, IN 47405, U.S.A. \\ Submitted 28 August 2014. Accepted 20 January 2015.
}

\begin{abstract}
The Pseudomonas syringae effector AvrB triggers a hypersensitive resistance response in Arabidopsis and soybean plants expressing the disease resistance $(R)$ proteins RPM1 and Rpg1b, respectively. In Arabidopsis, AvrB induces RPM1-interacting protein kinase (RIPK) to phosphorylate a disease regulator known as RIN4, which subsequently activates RPM1-mediated defenses. Here, we show that AvrPphB can suppress activation of RPM1 by AvrB and this suppression is correlated with the cleavage of RIPK by AvrPphB. Significantly, AvrPphB does not suppress activation of RPM1 by AvrRpm1, suggesting that RIPK is not required for AvrRpm1-induced modification of RIN4. This observation indicates that AvrB and AvrRpm1 recognition is mediated by different mechanisms in Arabidopsis, despite their recognition being determined by a single $\mathbf{R}$ protein. Moreover, AvrB recognition but not AvrRpm1 recognition is suppressed by AvrPphB in soybean, suggesting that AvrB recognition requires a similar molecular mechanism in soybean and Arabidopsis. In support of this, we found that phosphodeficient mutations in the soybean GmRIN4a and GmRIN4b proteins are sufficient to block Rpg1b-mediated hypersensitive response in transient assays in Nicotiana glutinosa. Taken together, our results indicate that AvrB and AvrPphB target a conserved defense signaling pathway in Arabidopsis and soybean that includes RIPK and RIN4.
\end{abstract}

Most plant pathogens express a suite of secreted effector proteins that enable them to infect their hosts. Many of these effectors have been shown to target components of pathogenassociated molecular pattern-triggered immunity (PTI) (Chen et al. 2004; He et al. 2006; Zhang et al. 2010). By shutting down these basal defenses, pathogens ensure that the host remains susceptible to colonization. But, simply acquiring as many effectors as possible is not the most effective strategy for pathogen success, because effectors tend to be a double-edged sword, promoting bacterial growth in susceptible hosts while betraying the pathogen in resistant hosts by triggering activation of corresponding resistance $(\mathrm{R})$ proteins (Block and Alfano 2011; Zhang and Zhou 2010). R proteins become activated in the presence of specific effectors, turning on a defense response

Current address for A. R. Russell: Department of Biology, Northern State University, Aberdeen, SD 57401, U.S.A.

Corresponding author: R. W. Innes; E-mail: rinnes@indiana.edu

*The $\boldsymbol{e}$-Xtra logo stands for "electronic extra" and indicates that one supplementary figure is published online.

(C) 2015 The American Phytopathological Society known as effector-triggered immunity (ETI) (Jones and Dangl 2006). ETI reactivates basal defenses that may be suppressed by the pathogen and induces a localized programmed cell death referred to as a hypersensitive response (HR).

There are currently 64 confirmed or predicted effector families employed by the various pathovars of Pseudomonas syringae, with any one strain harboring as many as 40 individual effectors (Schechter et al. 2004). If just one of these effectors activates an R protein, ETI is initiated and pathogen growth is limited. Once ETI is activated, it essentially nullifies any virulence benefit that the other effectors offer the bacteria. Therefore, it would be valuable for pathogen success if some effectors could also block activation of specific $\mathrm{R}$ proteins to prevent inadvertent stimulation of ETI. Indeed, several $P$. syringae effectors have been shown to specifically suppress recognition of other $P$. syringae effectors (Mackey et al. 2003; Rosebrock et al. 2007; Tsiamis et al. 2000).

A specific example of effector-mediated suppression of ETI is resistance mediated by the Arabidopsis R protein RPM1 (resistance to $P$. syringae pv. maculicola protein 1), which confers recognition of two P. syringae effectors, AvrB and AvrRpm1 (Bisgrove et al. 1994). RPM1 monitors disease regulator RIN4 (RPM1-interacting protein 4) and becomes activated when AvrB and AvrRpm1 induce phosphorylation of RIN4 (Mackey et al. 2002). AvrB and AvrRpm1 recognition can be suppressed, however, by the $P$. syringae effector AvrRpt2 (Mackey et al. 2002), which is a cysteine protease that cleaves RIN4 at two locations, leading to its rapid degradation (Axtell and Staskawicz 2003; Axtell et al. 2003; Mackey et al. 2003).

Neither AvrB nor AvrRpm1 contain a typical kinase domain, indicating that a host kinase may be required for RIN4 phosphorylation. Consistent with this view, expression of the Arabidopsis RPM1-interacting protein kinase (RIPK) is activated in the presence of AvrB and AvrRpm1 (Liu et al. 2011). Yet, a ripk knockout mutant is impaired in AvrB-triggered resistance but not AvrRpm1-triggered resistance (Liu et al. 2011). Regardless, RPM1 is presumed to detect AvrB- and AvrRpm1mediated phosphorylation of threonine 166, since phosphomimic mutations (T166D) at this residue trigger RPM1-dependent HR in the absence of either effector (Chung et al. 2011; Liu et al. 2011).

Plants from different species can often detect the same pathogen effectors. For instance, soybean cultivars can recognize AvrB and AvrRpm1 just like Arabidopsis; however, resistance to these effectors in soybean is conferred by two independent $\mathrm{R}$ proteins, Rpg1b and Rpg1r, respectively (Ashfield et al. 1995), rather than a single $\mathrm{R}$ protein, as is the case in Arabidopsis (Bisgrove et al. 1994). Such examples imply conservation of $R$ genes over long evolutionary periods or, alternatively, convergent 
evolution of recognition specificities among $R$ genes in different species. In this case, Rpglb and Rpglr are not orthologous with Arabidopsis RPM1, suggesting convergent evolution of AvrB and AvrRpm1 recognition mechanisms (Ashfield et al. 2004, 2014).

Soybean has four RIN4 homologs designated GmRIN4a to GmRIN4d. Coimmunoprecipitation and yeast two-hybrid analysis demonstrated that AvrB interacts with all four GmRIN4 homologs, while Rpg1b interacts with GmRIN4b to GmRIN4d but not GmRIN4a (Selote and Kachroo 2010). It is likely that at least one of these is required for AvrB and AvrRpm1 recognition in soybean, since AvrRpt2, which cleaves a conserved motif in these homologs, can partially block Rpg1b- and Rpg1r-mediated resistance (Ashfield et al. 2004, 2014; Kessens et al. 2014). Indeed, transient expression of a GmRIN4b phosphomimic mutant was sufficient to activate Rpg1b-dependent resistance (Selote et al. 2013), while Rpg1-dependent resistance is suppressed by virusinduced silencing of RIN4a or RIN4b (Selote et al. 2010). It is not well understood, however, how RPM1 can recognize both AvrB and AvrRpm1, while Rpg1b and Rpg1r recognize them individually.

Another effector secreted by certain $P$. syringae strains is a cysteine protease called AvrPphB. This effector recognizes and cleaves a conserved motif shared by Arabidopsis proteins from subfamily VII of the receptor-like cytoplasmic protein kinases (RLCK) (Shao et al. 2003; Zhang et al. 2010). Subfamily

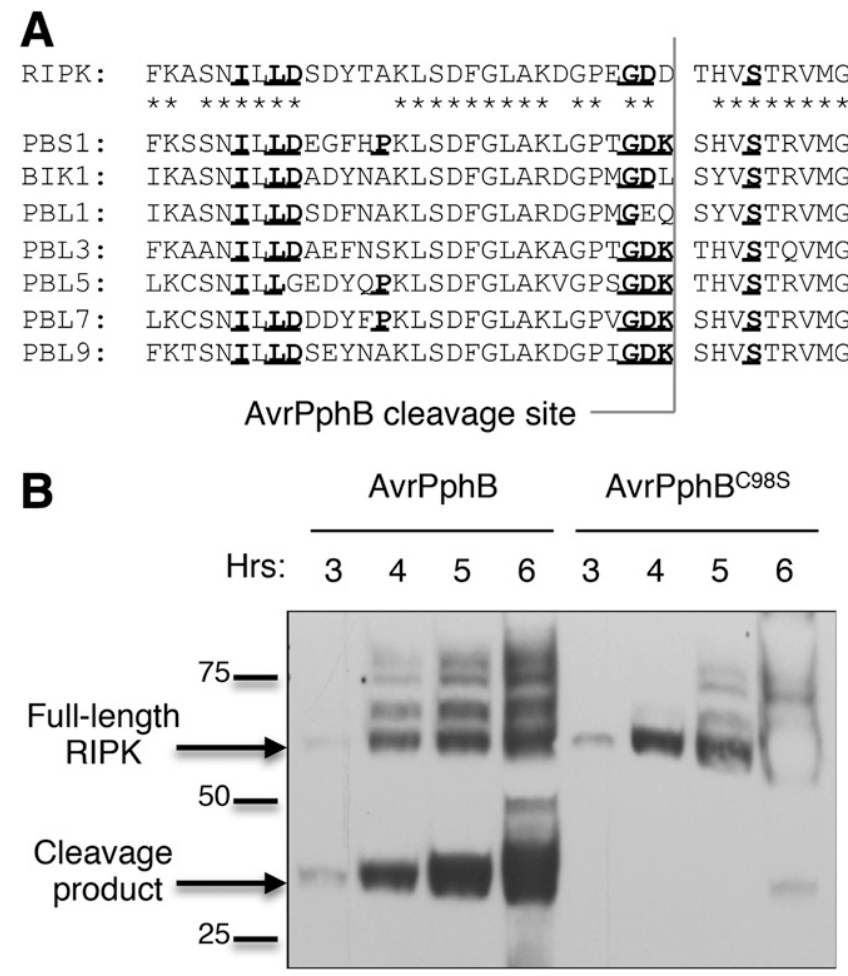

Fig. 1. AvrPphB cleaves RPM1-interacting protein kinase (RIPK). A, A short segment of amino acid sequence surrounding the AvrPphB cleavage site is shown for RIPK and seven PBS1-Like (PBL) kinase family members. Putative residues involved in AvrPphB recognition are in bold and underlined. Stars indicate residues conserved between RIPK and PBS1. The AvrPphB cleavage site is indicated following the GDK motif important for PBS1 cleavage. B, pTA7002::RIPK::HA::StrepII was coexpressed with pTA7002::avrPphB or pTA7002::avrPphB ${ }^{\text {98S }}$ by transient transformation of Nicotiana benthamiana. Protein expression was induced $40 \mathrm{~h}$ after injection by applying $50 \mu \mathrm{M}$ dexamethisone. Leaves were harvested 3 to $6 \mathrm{~h}$ after induction and protein was extracted for subsequent analysis. Total protein was separated by sodium dodecyl sulfate-polyacrylamide gel electrophoresis and was detected with antihemagglutinin horseradish peroxidase (anti-HA-HRP) antibody. Expected sizes: RIPK::HA::StrepII, approximtaely $55 \mathrm{kDa}$; C-terminal cleavage product, approximately $30 \mathrm{kDa}$.
VII includes important regulators of PTI and ETI, including BIK1 (Botrytis-induced kinase 1) and PBS1 (AvrPphB-susceptible 1). Cleavage of these proteins has been shown to suppress host defense responses such as transcription of FRK1 and callose deposition (Zhang et al. 2010). Hosts encoding the R protein RPS5 can recognize PBS1 cleavage and activate ETI, suppressing the growth of AvrPphB-expressing $P$. syringae strains (Ade et al. 2007; Shao et al. 2003; Simonich and Innes 1995).

The goal of our study was to investigate the capability of AvrPphB to enhance $P$. syringae growth by suppressing PTI and ETI. Cleavage of host kinases by AvrPphB likely disrupts defense signaling activated by numerous bacterial elicitor molecules. Zhang et al. (2010) recently reported that AvrPphB can suppress basal defenses in the host. We have extended their findings by demonstrating that AvrPphB-dependent PTI disruption enhances the growth of a hypovirulent $P$. syringae strain lacking a functional type III secretion system (T3SS). Moreover, we found that AvrPphB can block recognition of AvrB by cleaving the Arabidopsis kinase RIPK. This virulence phenotype is conserved in soybean cultivars encoding $\mathrm{R}$ protein Rpg1b, indicating a mechanism by which AvrPphB can mask the presence of AvrB in two different plant families.

\section{RESULTS}

AvrPphB cleaves the Arabidopsis kinase RIPK.

RIPK belongs to the same subfamily of RLCK as PBS1 and BIK1 (Liu et al. 2011; Swiderski and Innes 2001; Zhang et al. 2010), but its ability to be cleaved by AvrPphB has not previously been reported. RIPK displays high sequence similarity to PBS1 and other PBS1-like (PBL) family members surrounding the AvrPphB cleavage site (Fig. 1A). We hypothesized that RIPK might be a substrate of AvrPphB and that cleavage by AvrPphB might block recognition of AvrB and, thus, prevent RPM1 activation. To test this, we transiently expressed RIPK together with AvrPphB in Nicotiana benthamiana and checked for cleavage by immunoblot. Consistent with our hypothesis, PBS1 and RIPK were both cleaved when coexpressed with AvrPphB but not when coexpressed with the enzymatically-impaired mutant $\mathrm{AvrPphB}^{\mathrm{C} 98 \mathrm{~S}}$ (Fig. 1B). Therefore, RIPK, like PBS1, is efficiently cleaved by AvrPphB in vivo, providing a possible mechanism for suppression of RPM1 activation.

\section{AvrPphB enhances growth of DC3000(avrB) by suppressing RPM1 activation.}

To see if AvrPphB-induced RIPK cleavage affects RPM1 function, we examined whether AvrPphB could suppress the HR induced by AvrB when both effectors were delivered by the same $P$. syringae strain. We observed a reduction in the severity of leaf collapse induced by $P$. syringae expressing AvrB plus AvrPphB compared with that induced by $P$. syringae expressing AvrB alone; however, this reduction was modest and somewhat variable (data not shown). To assess more quantitatively whether AvrPphB could suppress AvrB recognition, we measured bacterial growth of $P$. syringae pv. tomato DC3000(avrB) or $P$. syringae pv. tomato DC3000(empty vector; EV) on transgenic rps5 Arabidopsis plants expressing AvrPphB under an estradiolinducible promoter. Treatment of two-week-old seedlings with estradiol induced robust AvrPphB expression (Supplementary Fig. 1). Importantly, a growth curve on five-week-old plants showed an approximately $1 \log$ increase in growth of $P$. syringae pv. tomato DC3000( $a v r B)$ in plants treated with estradiol compared with untreated plants (Fig. 2A). This suggests that transgenic expression of AvrPphB is sufficient to enhance the growth of a bacterial strain carrying AvrB on plants that would normally be resistant. 
The growth-curve analysis described above was dependent upon ectopic overexpression of AvrPphB in host tissues. The level of $\mathrm{AvrPphB}$ expression in these experiments was likely higher than that seen in a typical $P$. syringae infection. To substantiate the biological significance of these findings and confirm that AvrPphB suppresses RPM1 signaling under natural expression levels, we generated a DC3000 strain that coexpresses AvrB and AvrPphB on separate plasmids. This strain allowed us to investigate the virulence effect of AvrPphB after bacterial delivery via the type III secretion system (T3SS). Consistent with our transgenic data, $P$. syringae pv. tomato DC3000 expressing $a v r B$ and $a v r P p h B$ grew a full log higher than $P$. syringae pv. tomato DC3000 expressing $a v r B$ and EV (Fig. 2B) on Arabidopsis plants expressing RPM1. Thus, delivery of AvrPphB directly from the bacterial T3SS is sufficient to enhance the growth of strains expressing $a v r B$.

AvrPphB may be promoting the virulence of $a v r B$-expressing strains by blocking PTI. As mentioned above, AvrPphB cleaves multiple PBL protein kinases, many of which contribute to PTI signaling. Cleavage of these targets perturbs many basal responses, such as callose deposition, $\mathrm{H}_{2} \mathrm{O}_{2}$ production, and defense gene expression (Zhang et al. 2010). To see if this correlates with an
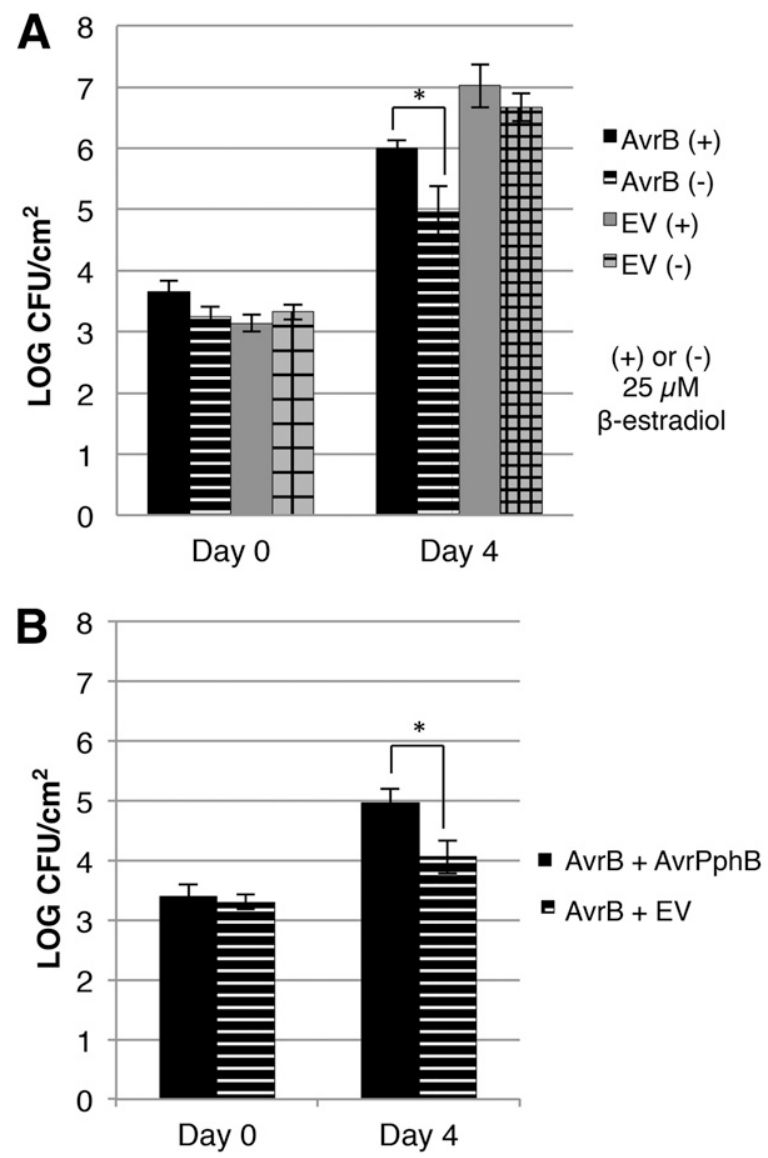

Fig. 2. AvrPphB suppresses recognition of AvrB in Arabidopsis. A, Transgenic expression and $\mathbf{B}$, bacterial delivery of AvrPphB was tested for effect on resistance to AvrB. A, Pseudomonas syringae pv. tomato $\mathrm{DC} 3000(a v r B)$ or empty vector (EV) were inoculated onto transgenic rps5 Arabidopsis expressing avrPphB under a $\beta$-estradiol-inducible promoter. Transgene expression was induced by spraying plants with $25 \mu \mathrm{M} \beta$-estradiol $6 \mathrm{~h}$ before bacterial inoculation and once a day for the duration of the experiment. B, P. syringae pv. tomato DC3000 $(a v r B+a v r P p h B)$ or $(a v r B+E V)$ was inoculated onto the Arabidopsis rps 5 mutant line. Five-week-old plants were infiltrated with a bacterial suspension at an optical density at $600 \mathrm{~nm}=$ 0.0005 . Bacterial titers were determined immediately after inoculation and 4 days later. Error bars represent \pm 1 standard deviation. Growth curves were repeated twice with similar results. *Student's $t$ test $P<0.05$. increase in bacterial growth, we inoculated the $a v r P p h B$ transgenic line with a $P$. syringae pv. tomato DC3000 hrcC mutant strain and monitored growth in the presence or absence of $a v r P p h B$ expression. The $h r c C$ gene is necessary for a functional T3SS (Charkowski et al. 1997; Deng et al. 1998; Roine et al. 1997). Consequently, this strain is unable to colonize the host due to its inability to deliver type III effectors into host cells. P. syringae pv. tomato DC3000 hrcC growth increased a half log in the presence of transgenic avrPphB expression (Fig. 3), indicating that AvrPphB does, indeed, suppress PTI enough to partially compensate for a T3SS deficiency.

Since AvrPphB suppresses PTI, it was not clear from the above experiments whether enhanced $P$. syringae pv. tomato DC3000( $a v r B)$ growth on the $a v r P p h B$ transgenic line was due to diminished RPM1 function or simply weakened basal defenses. To distinguish between these scenarios, we compared the growth of $P$. syringae pv. tomato DC3000(avrB) and $P$. syringae pv. tomato DC3000(avrRpt2) on the avrPphB transgenic line. AvrRpt 2 is recognized by another R protein, RPS2, which has not been shown to require RIPK or any other PBL. Thus, RPS2mediated resistance should be unaffected by AvrPphB expression and any increase in $P$. syringae pv. tomato DC3000(avrRpt2) growth would simply be due to weakened basal defenses. Our results showed a negligible increase in $P$. syringae pv. tomato DC3000(avrRpt2) growth on plants expressing avrPphB (Fig. 4), but this effect was not statistically significant. We obtained a similar result with a more stringent control comparing the growth of $P$. syringae pv. tomato $\mathrm{DC} 3000(a v r B)$ and $P$. syringae pv. tomato DC3000(avrRpml) on the avrPphB transgenic line. Because AvrRpm1-triggered resistance is dependent on RPM1 but independent of RIPK (Liu et al. 2011), any enhanced growth of $P$. syringae pv. tomato $\mathrm{DC} 3000($ avrRpml) in the presence of AvrPphB should be due to weakened PTI. If AvrPphB has an equal growth effect on $P$. syringae pv. tomato $\mathrm{DC} 3000(a v r B)$ and $P$. syringae pv. tomato DC3000(avrRpml), it is likely due to a role in blocking PTI and not ETI. But, $P$. syringae pv. tomato DC3000(avrRpm1) growth was not enhanced by avrPphB expression, suggesting that the enhanced growth of $P$. syringae pv. tomato $\mathrm{DC} 3000($ avrB $)$ is due to suppression of AvrB recognition

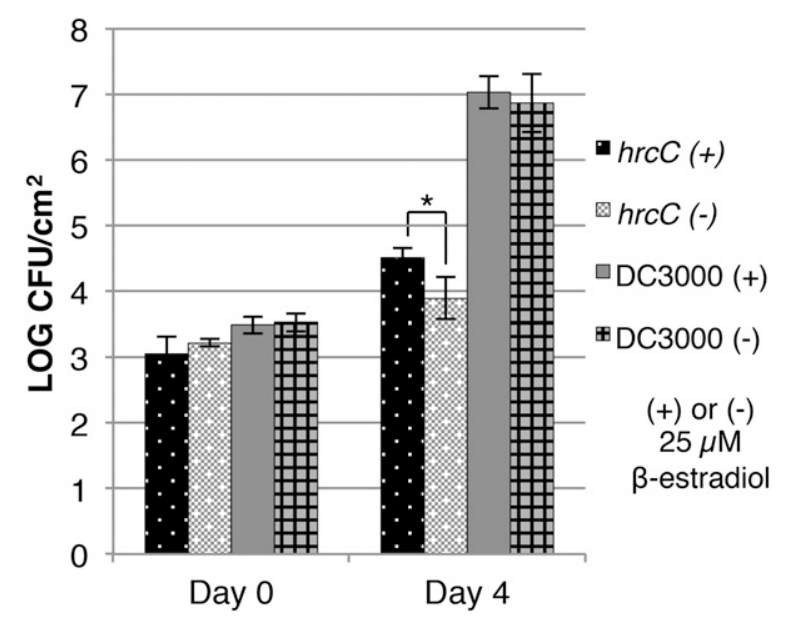

Fig. 3. Transgenic expression of AvrPphB enhances growth of the hypovirulent hrcC mutant. The indicated Pseudomonas syringae pv. tomato strains were inoculated onto transgenic rps5 Arabidopsis expressing avrPphB under an estradiol-inducible promoter. Five week old plants were infiltrated with a bacterial suspension at an optical density at $600 \mathrm{~nm}=0.0005$. Transgene expression was induced by spraying plants with $25 \mu \mathrm{M} \beta$-estradiol $6 \mathrm{~h}$ before bacterial inoculation and once a day for the duration of the experiment. Bacterial titers were determined immediately after inoculation and 4 days later. Error bars represent $+/-$ one standard deviation. The growth curve was repeated twice with similar results. *Student's $t$ test $P<0.05$. 
rather than general suppression of PTI (Fig. 5A). Furthermore, codelivery of AvrRpm1 and AvrPphB via the T3SS gave similar results, as no significant growth difference was observed between $P$. syringae pv. tomato DC3000(avrRpm1 + avrPphB) and $P$. syringae pv. tomato DC3000(avrRpm $1+\mathrm{EV})$ (Fig. 5B). These data, combined with our transgenic analysis, demonstrate that $a v r P p h B$ expression can mask the presence of AvrB in hosts that would normally activate AvrB-triggered ETI.

\section{AvrPphB suppresses resistance mediated by the soybean $R p g 1 b$ gene.}

AvrB can also induce ETI in soybean cultivars that express the resistance protein Rpg1b (Ashfield et al. 1995). To see if AvrPphB could also enhance growth of an AvrB-carrying strain in soybean, we screened 35 accessions from the United States Department of Agriculture soybean germplasm collection for resistance to $P$. syringae pv. glycinea race 4 carrying avrB (PsgR4[avrB]) and susceptibility to $P s g R 4(a v r P p h B)$, using visual assessment of disease symptoms following a dip-inoculation. Three lines from this screen ('Gnome', 'Hardee', and 'Harcor') were chosen for growth-curve analysis because they exhibited apparent disease symptoms (chlorotic, water-soaked lesions) to $P s g R 4(a v r P p h B)$ and no visible symptoms in response to PsgR4(avrB) (Fig. 6A). The selected lines were dip-inoculated with $P s g R 4(a v r B)$ and $P s g R 4(a v r P p h B)$, and bacterial growth was assessed after 4 days to confirm the results of the visual screen. All three cultivars were resistant to $P s g R 4(\operatorname{avrB})$. However, cultivars Gnome and Hardee were also resistant to PsgR4(avrPphB), despite showing some disease symptoms in our visual screen (Fig. 6B). Only 'Harcor' demonstrated the right combination of resistance to $P s g R 4(a v r B)$ and full susceptibility to PsgR4(avrPphB).

To evaluate the effect of AvrPphB on AvrB-triggered resistance in 'Harcor', we generated $P s g R 4$ strains expressing $a v r B$ together with $a v r P p h B$ or the enzymatically-impaired mutant $\operatorname{avrPph} B^{C 98 S}$. These strains were vacuum-infiltrated into 'Harcor' and bacterial growth was monitored for 4 days.

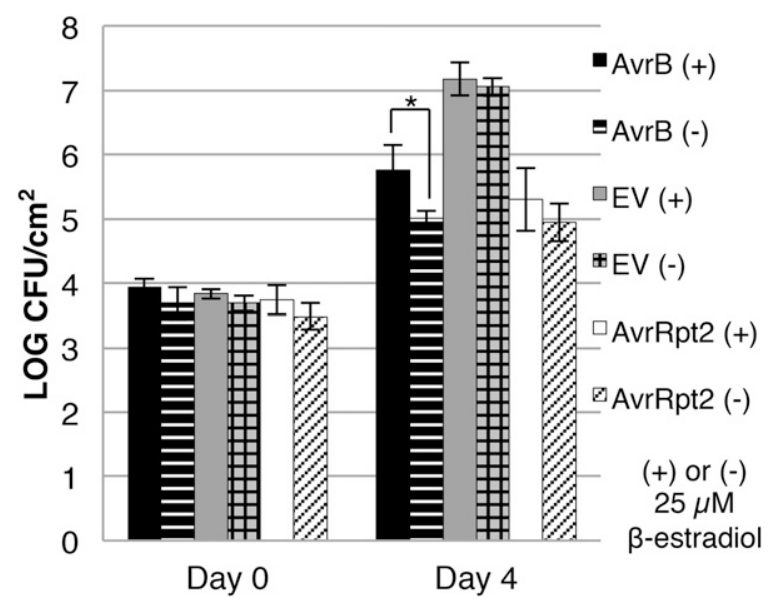

Fig. 4. Transgenic expression of AvrPphB does not suppress recognition of AvrRpt2. Pseudomonas syringae pv. tomato DC3000 (avrRpt2), (avrB), or empty vector (EV) were inoculated onto transgenic rpm1 Arabidopsis expressing $a v r P p h B$ under an estradiol-inducible promoter. Five-week-old plants were infiltrated with a bacterial suspension at an optical density at $600 \mathrm{~nm}=0.0005$. Transgene expression was induced by spraying plants with $25 \mu \mathrm{M} \beta$-estradiol $6 \mathrm{~h}$ before bacterial inoculation and once a day for the duration of the experiment. Bacterial titers were determined immediately after inoculation and 4 days later. Error bars represent \pm 1 standard deviation. The growth curve was repeated twice with similar results. *Student's $t$ test $P<0.05$.
The control strains $P s g R 4(a v r P p h B)$ and $P s g R 4(a v r B:: \Omega)$ grew to a high titer, confirming that 'Harcor' supports substantial growth of these strains and does not have any resistance to AvrPphB (Fig. 7A). Furthermore, AvrPphB partially suppressed AvrB-triggered resistance with $P s g R 4(a v r B+a v r P p h B)$, growing to significantly higher levels than $\operatorname{PsgR} 4\left(a v r B+A v r P p h B{ }^{C 98 S}\right)$. These results show that AvrPphB can suppress recognition of AvrB in both Arabidopsis and soybean and suggest that soybean employs a RIPK homolog in the recognition of AvrB.

'Harcor' and other soybean cultivars that are resistant to AvrB express R protein Rpg1b. But, resistance to AvrRpm1 is dependent upon a separate $\mathrm{R}$ protein, $\mathrm{Rpg} 1 \mathrm{r}$. To determine if AvrPphB-dependent growth enhancement in soybean is due specifically to Rpg1b impairment, we compared the growth of PsgR4(avrRpml + avrPphB) and PsgR4(avrRpml + EV) on cultivar Acme, which is susceptible to AvrPphB but expresses Rpg1r for recognition of AvrRpm1. Importantly, we did not observe any growth difference between the two strains (Fig. 7B),
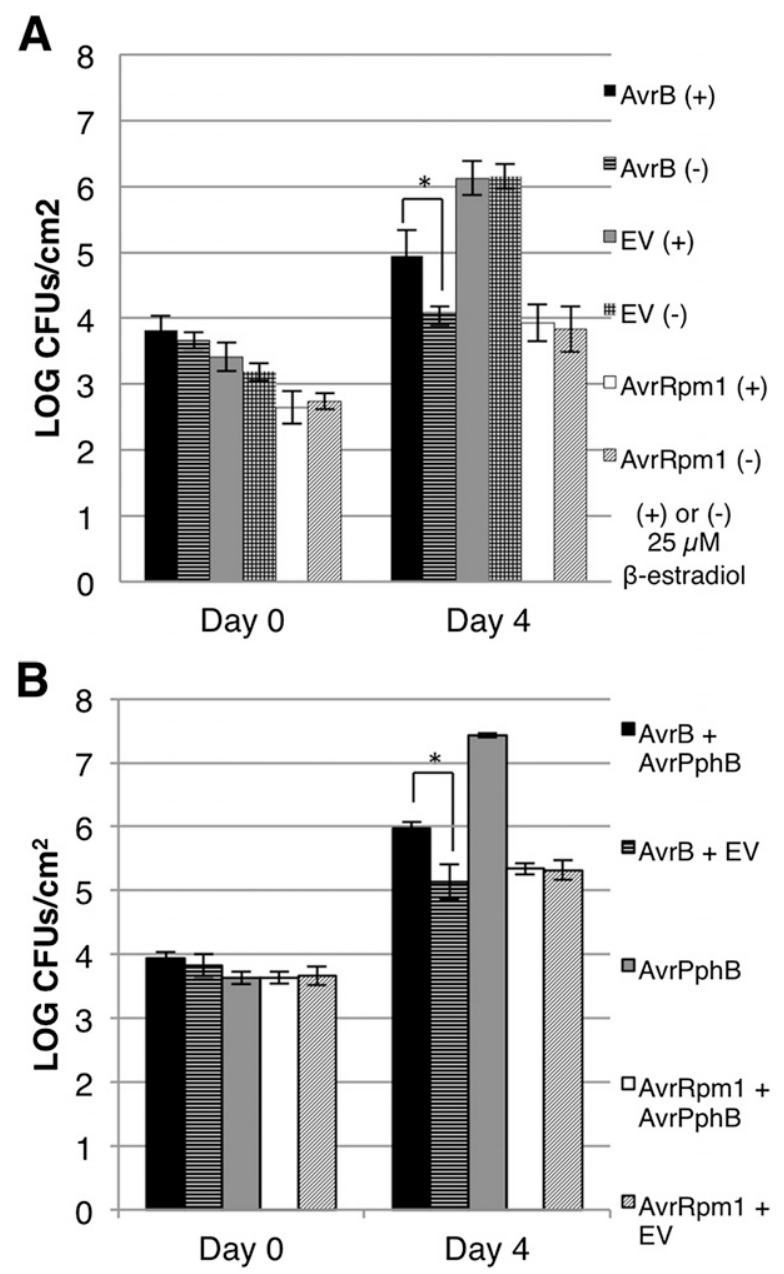

Fig. 5. AvrPphB does not suppress recognition of AvrRpm1. A, Transgenic expression and $\mathbf{B}$, bacterial delivery of AvrPphB was tested for effect on resistance to Pseudomonas syringae pv. tomato DC3000(avrRpm1). A, The indicated $P$. syringae pv. tomato DC3000 strains were inoculated onto transgenic rpm1 Arabidopsis expressing avrPphB under a $\beta$-estradiol-inducible promoter. Transgene expression was induced by spraying plants with $25 \mu \mathrm{M}$ $\beta$-estradiol $6 \mathrm{~h}$ before bacterial inoculation and once a day for the duration of the experiment. B, The indicated P. syringae pv. tomato DC3000 strains were inoculated onto the Arabidopsis rps5 mutant line. Five-week-old plants were infiltrated with a bacterial suspension at an optical density at $600 \mathrm{~nm}=0.0005$. Bacterial titers were determined immediately after inoculation and 4 days later. Error bars represent \pm 1 standard deviation. Growth curves were repeated twice with similar results. *Student's $t$ test $P<0.05$. 
suggesting that AvrPphB does not impair Rpg1r function. Thus, our results suggest that AvrPphB blocks AvrB recognition in soybean through a mechanism that specifically disrupts Rpg1bmediated resistance and not resistance mediated by other $\mathrm{R}$ proteins.

\section{Phosphodeficient GmRIN4 mutants block Rpg1b activation.}

The ability of AvrPphB to suppress AvrB recognition in soybean suggests that this recognition may involve a RIPK homolog in soybean. Arabidopsis RIPK has been shown to phosphorylate Arabidopsis RIN4 at threonine 166, and phosphomimic mutations at this residue are sufficient for the activation of RPM1 (Chung et al. 2011; Liu et al. 2011). Significantly, threonine 166 is conserved in all four soybean RIN4 homologs, and Rpg1bmediated resistance requires GmRIN4a and GmRIN4b (Selote and Kachroo 2010). If this conserved threonine is necessary for Rpg1b function, mutating it to a nonphosphorylatable amino acid should prevent Rpg1b activation in the presence of its corresponding effector. To test this, we used site-directed mutagenesis to substitute an alanine residue for the conserved threonine in GmRIN4a and GmRIN4b, generating the following mutant alleles in the dexamethasone-inducible expression vector pTA7002: GmRIN4 ${ }^{\mathrm{T} 198 \mathrm{~A}}$ and GmRIN4b ${ }^{\mathrm{T} 198 \mathrm{~A}}$. Next, these constructs were transiently expressed in Nicotiana glutinosa to evaluate their effect on Rpg1b activation. In this system, Rpg1b elicits a strong HR when coexpressed with AvrB and GmRIN4a or GmRIN4b (Kessens et al. 2014). Substituting either wild-type GmRIN4 allele with a T198A phosphodeficient mutant allele blocked HR (Fig. 8). This result shows that T198 is necessary for Rpg1b-mediated resistance and supports our model that AvrPphB can suppress $\mathrm{R}$ protein activation in the presence of AvrB by blocking modification of RIN4 proteins in both Arabidopsis and soybean (Fig. 9).

\section{DISCUSSION}

The $P$. syringae effector AvrPphB is a cysteine protease that promotes bacterial growth by cleaving host proteins that share a conserved recognition motif (Shao et al. 2003). AvrPphB specifically targets the RLCK VII family of protein kinases, which includes PBS1 and BIK1 (Zhang et al. 2010). This kinase family has been shown to promote PTI-associated responses such as callose deposition, $\mathrm{H}_{2} \mathrm{O}_{2}$ production, and FRK1 expression (Zhang et al. 2010). We found that suppression of these basal resistance outputs correlates with an overall reduction in PTI that enhances the growth of a T3SS-deficient $P$. syringae hrcC mutant. Remarkably, ectopic AvrPphB expression in Arabidopsis can overcome a T3SS deficiency and enhance the growth of a strain that is normally unable to multiply in Arabidopsis on its own. This result supports previous reports that one of the virulence functions of AvrPphB is to suppress PTI through cleavage of PBL kinases (Zhang et al. 2010).

The focus of our study was to determine whether AvrPphB can also promote bacterial growth by suppressing ETI in certain host genotypes. This hypothesis was derived from the fact that recognition of the $P$. syringae effector AvrB requires a member of the PBL kinase family called RIPK (Liu et al. 2011). Upon delivery into host cells, AvrB recruits RIPK to phosphorylate RIN4. Resistant hosts sense this modification via RPM1, which is activated by perturbations to RIN4. Consequently, RPM1 activation triggers a classic HR that limits pathogen growth in the presence of AvrB. Because AvrPphB efficiently cleaves RIPK in planta (Fig. 1B), we hypothesized that AvrPphB might block RPM1-triggered ETI by cleaving RIPK to prevent RIN4 phosphorylation (Fig. 9). To support this, we showed that AvrPphB expressed transiently in the plant or delivered directly from the bacteria enhances growth of $P$. syringae pv. tomato strains carrying avrB in Arabidopsis plants expressing RPM1. Significantly, AvrPphB did not suppress recognition of either AvrRpt 2 or AvrRpm1 (Fig. 4 and 5). These results are consistent with a previously published report showing that AvrRpm1triggered resistance does not require RIPK (Liu et al. 2011) and indicate that AvrPphB likely suppresses AvrB recognition by interfering with RIPK-mediated activation of RPM1. These observations raise the question of how AvrRpm1 activates RPM1. Prior work has indicated that RIN4 becomes phosphorylated in the presence of AvrRpm1 (Mackey et al. 2002). Our data suggest that this must occur via a direct enzymatic activity of AvrRpm1 or, else, via a host kinase not cleaved by AvrPphB.

The suppression of AvrB-triggered resistance by AvrPphB appears to be stronger than that observed in a ripk null mutant. Liu et al. (2011) showed that a ripk knockout mutation in Arabidopsis increases the growth of $P$. syringae DC3000(avrB) approximately fourfold, whereas we observed that transgenic expression of AvrPphB in Arabidopsis increased the growth of DC3000 ( $a v r B)$ by 10-fold (Fig. 2A). These data support a model proposed by Liu et al. (2011) in which RIN4 is phosphorylated by multiple members of the RIPK family (RLCK VII). Because AvrPphB can cleave the majority of RLCK VII family members (Zhang et al. 2010), it would be expected to have a stronger impact on AvrB recognition than would mutation of RIPK alone. Consistent with this model, mutation of $R I P K$ only partially reduces AvrB-induced phosphorylation of RIN4 (Liu et al. 2011).

Host range and specificity are dynamic characteristics that shift as pathogen and host genomes evolve. Since horizontal transfer of effector genes between bacterial strains is a frequent occurrence (El Yacoubi et al. 2007; Ma et al. 2006; McCann and Guttman 2008), acquisition of a new effector changes a pathogen's ability to manipulate host physiology. An interesting aspect of this is whether it benefits pathogens to maintain certain combinations of effectors. Our results show that one virulence mechanism of AvrPphB is to mask the presence of AvrB. Host genotypes that were once able to detect AvrB through RPM1 no longer recognize its presence because AvrPphB has removed a crucial cog in the RPM1 activation pathway. This relationship is similar to that observed with AvrRpt 2 and AvrB. AvrRpt 2 can also block AvrB recognition by degradation of RIN4, which is another necessary component of RPM1 activation (Axtell and Staskawicz 2003; Mackey et al. 2002, 2003). Since RPM1 is prevalent in Arabidopsis accessions, it might be evolutionarily favorable for $P$. syringae strains that express AvrB to also express AvrPphB or AvrRpt2. If this were the case, one would expect to find these effectors jointly preserved in $P$. syringae genomes. Of the 19 currently published $P$. syringae genomes, seven encode AvrB, four encode a functional AvrPphB, and only one encodes AvrRpt2 (Baltrus et al. 2011). However, $a v r B$ is not present in the same genome as avrRpt2, while $a v r B$ is only present in one of the four genomes that contain $a v r P p h B$, the genome of $P$. syringae pv. actinidae (strain MAFF302091) (Baltrus et al. 2011), which is a pathogen of kiwifruit plants (Actinidia deliciosa). This comparative genomic analysis does not support the theory that AvrPphB's principle role is suppression of AvrB recognition. It is possible that these 19 genomes do not accurately reflect the true frequency of $a v r B$ and $a v r P p h B$ in the wild. More likely, however, the distribution of these effectors is limited by the frequency of $\mathrm{R}$ proteins that detect AvrPphB and AvrRpt2, which would offset any benefit these effectors have for the bacteria. For example, the Arabidopsis $\mathrm{R}$ proteins RPS5 and RPS2 confer resistance to AvrPphB and AvrRpt2, respectively, and many of the soybeans lines we tested 
recognized AvrPphB. Moreover, suppression of AvrB-induced resistance may be a secondary virulence function of AvrPphB, since it also enhances bacterial growth by suppressing PTI (Fig. 3).

Arabidopsis RPM1 is activated in the presence of both AvrB and AvrRpm1, while soybean utilizes two R proteins, Rpg1b and Rpg1r, to recognize each effector independently. Understanding the genetic and molecular basis for this divergence has been a long-standing pursuit of the Innes lab. Therefore, it was of interest to us to determine if AvrPphB also impacted $\mathrm{Rpg} 1 \mathrm{~b} / \mathrm{Rpg} 1 \mathrm{r}-$ mediated immunity in soybean. To this end, we identified 'Harcor' as a suitable soybean line to test AvrBtriggered defenses, as it was found to be susceptible to PsgR4 strains expressing $a v r P p h B$ but resistant to $a v r B$-expressing strains. Interestingly, bacterial delivery of AvrPphB enhanced the growth of $P s g R 4$ strains carrying $a v r B$ in 'Harcor'. Since AvrPphB expression also masks AvrB in this normally resistant soybean cultivar, at least part of AvrPphB's virulence function is conserved in soybean. In contrast, the growth of $P s g R 4$ strains carrying avrRpml was unaffected by $a v r P p h B$ expression, suggesting that an AvrPphB cleavage target is necessary for complete Rpglb-mediated immunity but not
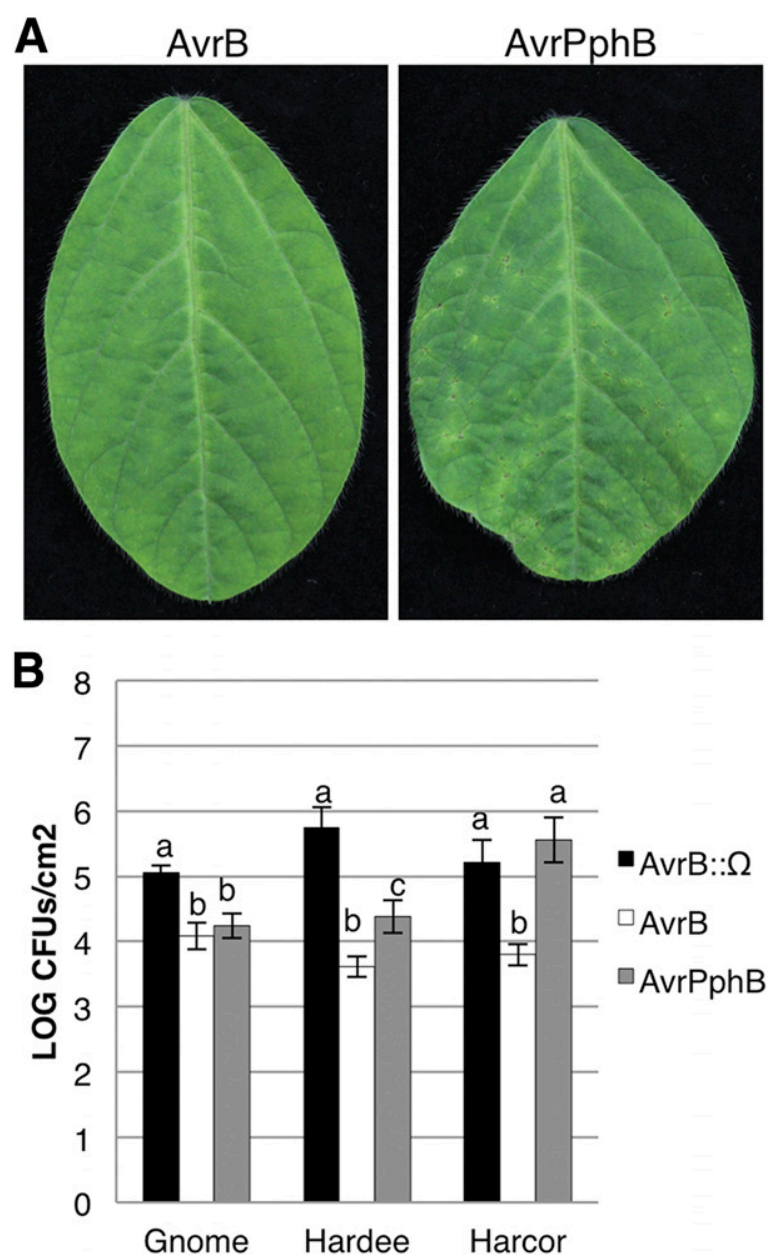

Fig. 6. Soybean cultivar Harcor is resistant to $P \operatorname{sgR} 4(\mathrm{avrB})$ and susceptible to $P s g R 4(a v r P p h B)$. A, Disease symptoms on unifoliate leaves of cultivar Harcor 10 days postinoculation with $P s g R 4$ expressing $a v r B$ or $a v r P p h B$. Similar symptoms were observed on cultivars Gnome and Hardee. B, Growth curve analyses. Two-week-old plants were dip-inoculated with the indicated Pseudomonas syringae pv. glycinea strains at an optical density at $600 \mathrm{~nm}=0.5$ Bacterial titers were determined 4 days later. Error bars represent \pm 1 standard deviation. Different letters indicate statistically significant differences (Student's $t$ test $P<0.05$ ).
Rpg1r-mediated immunity. This supports the notion that effector recognition is directly linked to effector activity. Indeed, AvrB mutants that lose virulence activity also lose the ability to trigger defenses in soybean and Arabidopsis alike (Ong and Innes 2006).

The Arabidopsis RIN4 residue T166 is crucial for RPM1 activation, since an alanine substitution at this residue blocks RPM1-mediated HR (Chung et al. 2011; Liu et al. 2011). Because the soybean GmRIN4 homologs share this conserved threonine, we wondered if Rpg1b might also require this residue for recognizing AvrB. While AvrB expression activated Rgp1bmediated HR when coexpressed with wild-type GmRIN4a, substitution of the phosphodeficient GmRIN4a ${ }^{\text {T198A }}$ or GmRIN4b ${ }^{\text {T198A }}$ allele abolished HR (Fig. 8). Our results thus define a point of convergence between RPM1- and Rpg1bmediated immunity, suggesting that modification of this residue is central to the biochemical detection of AvrB by plant surveillance systems. Therefore, despite RPM1 and Rpg1b having evolved the capacity to recognize AvrB independently (Ashfield et al. 2004), the molecular and biochemical requirements for AvrB recognition by each appear to be quite similar.
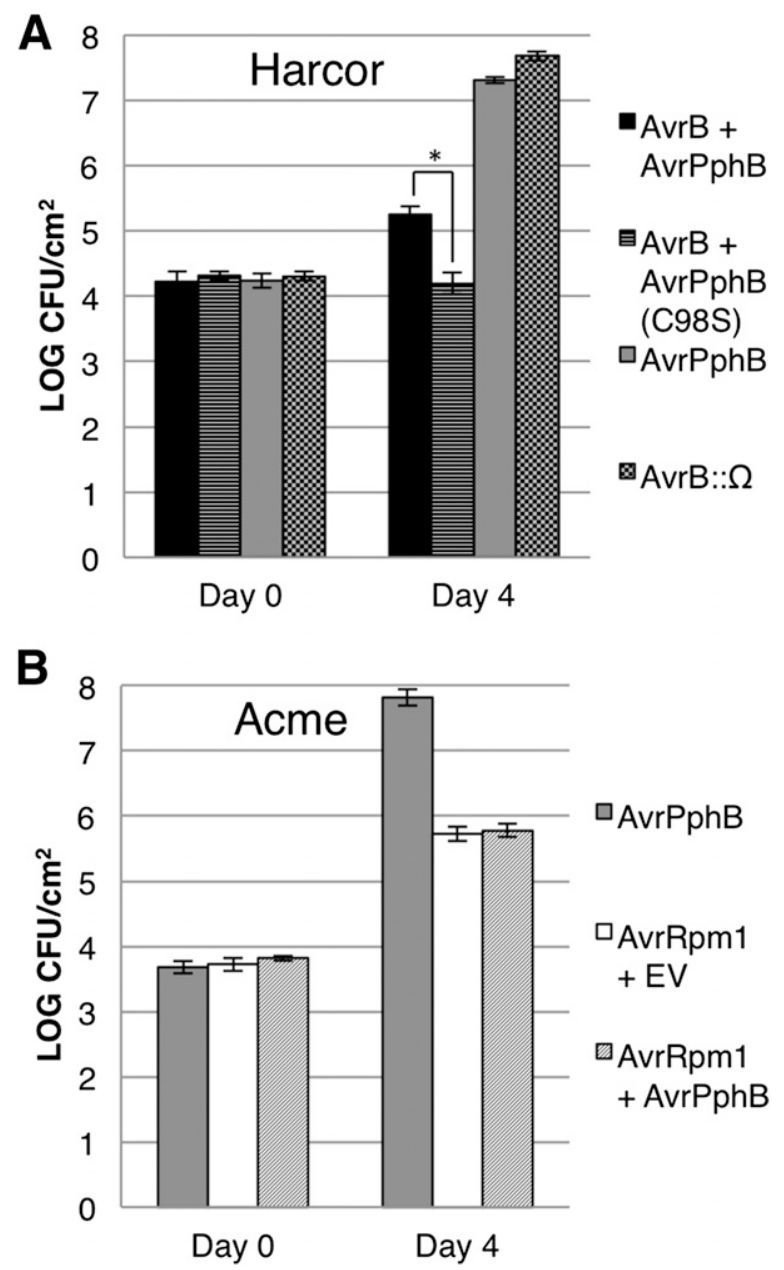

Fig. 7. Rpg1b- but not Rpg1r-mediated resistance is impaired in soybean by bacterially delivered AvrPphB. A, PsgR4 $(a v r B+a v r P p h B)$ or $(a v r B+$ avrPphB ${ }^{C 98 S}$ ) were inoculated onto soybean cultivar Harcor. B, PsgR4 (avrRpm1 + avrPphB) or (avrRpm1 + EV) were inoculated onto soybean cultivar Acme. Two-week-old plants were vacuum-infiltrated with a bacterial suspension at an optical density at $600 \mathrm{~nm}=0.0005$. Bacterial titers were determined immediately after inoculation and 4 days later. Error bars represent \pm 1 standard deviation. Growth curves were repeated twice with similar results. *Student's $t$ test $P<0.05$. 


\section{MATERIALS AND METHODS}

Plant lines and growth.

$N$. benthamiana seeds were sown in Metro-Mix 360 potting mixture (Sun Gro Horticulture) and were grown under a 9-h photoperiod at $22^{\circ} \mathrm{C}$. N. glutinosa seeds were sown in a $3: 1$ mixture of Metro-Mix 360 potting soil and expanded perlite and were grown for the first 10 to 14 days under a 16-h photoperiod and, then, transferred to a 9-h photoperiod. Arabidopsis seeds
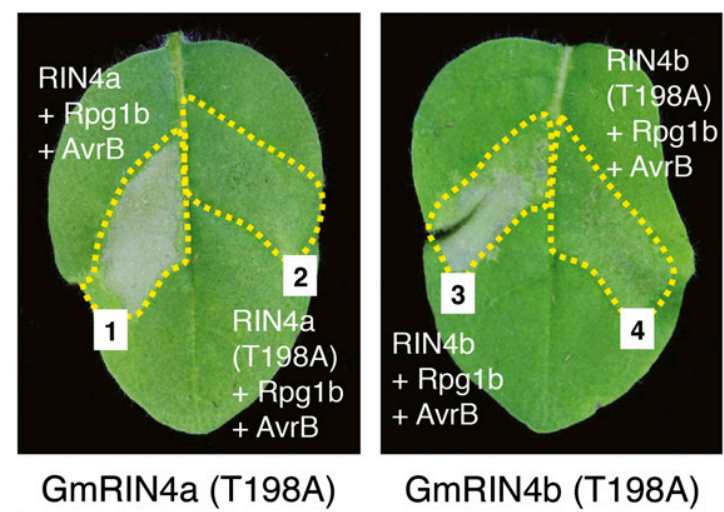

\begin{tabular}{|c|c|}
\hline \multicolumn{2}{|c|}{ GmRIN4b (T198A) } \\
\hline $\begin{array}{c}\text { Transgene } \\
\text { Combination }\end{array}$ & $\begin{array}{c}\text { HR } \\
\text { Observed }\end{array}$ \\
\hline 3 & $23 / 23$ \\
\hline 4 & $5 / 23$ \\
\hline
\end{tabular}

Fig. 8. Phosphodeficient mutations in GmRIN4 paralogs block activation of Rpg1b by AvrB. GmRIN4a and GmRIN4b as well as their phosphodeficient (T198A) derivatives were transiently expressed in Nicotiana glutinosa with Rpg1b and AvrB. Plants were sprayed with dexamethasone approximately $40 \mathrm{~h}$ after infiltration with Agrobacterium tumefaciens. Leaves were scored for the hypersensitive response approximately $30 \mathrm{~h}$ postinduction. Scores represent the number of leaves showing visible collapse out of the total number of leaves injected. The experiment was repeated twice for GmRIN4a and once for GmRIN4b with similar results. (ecotype Col-0 [rps5] and [rps5, pES-avrPphB]) (Qi et al. 2014) were sown in Metro-Mix 360 potting mixture, were stratified at $4^{\circ} \mathrm{C}$ for 3 to 4 days, and were grown under a 9-h photoperiod at $22^{\circ} \mathrm{C}$ for 6 to 8 weeks. All Nicotiana and Arabidopsis plants were grown in growth rooms under artificial light $\left(150 \mu \mathrm{Em}^{-2} \mathrm{~s}^{-1}\right)$. Soybean plants were sown in clay pots containing a compost/ Metro-Mix 360 (Sun Gro Horticulture)/vermiculite/perlite (4:2: $1: 1)$ mix supplemented with osmocote slow-release fertilizer and were grown in a growth chamber at $23^{\circ} \mathrm{C}$ under a 16 -h photoperiod. Pathogenicity tests on soybean were conducted 2 to 3 weeks after planting.

\section{Construction of protein expression vectors.}

The $a v r P p h B$ and protease inactive $a v r P p h B(\mathrm{C} 98 \mathrm{~S})$ pTA7002 constructs were generated as previously described by Shao et al. (2003). The Rpglb, avrB, GmRIN4a, and GmRIN4b genes were cloned into pTA7002 (Aoyama and Chua 1997) as described by Kessens et al. (2014), with the GmRIN4a and GmRIN4b genes containing an N-terminal $5 \times-M y c$ epitope tag, while the $a v r B$ and Rpglb genes were untagged. Phosphodeficient substitutions (T198A) in the GmRIN4 constructs were generated using the QuickChange site-directed mutagenesis kit from Agilent Technologies, following the manufacture's protocol. The pTA7002::RIPK construct was generated as follows. RIPK was amplified from Arabidopsis cDNA with the following attB-containing primers: attB1-RIPK-F, 5'-GGG GAC AAG TTT GTA CAA AAA AGC AGG GCT ACA TGG CGG TGA AGA AGA AAG TTT CAT-3', and $a t t B 2-R I P K-R, 5^{\prime}$-GGG GAC AAC TTT GTATAG AAA AGT TGG GTG GTA CCG TTC CCC ACC TGC CTC A-3'. A strepIIHA Gateway insert was generated separately by amplifying the human influenza hemagglutinin (HA) sequence, using the following primers: attB1-StrepII-HA-F, 5'-GGG-GAC-AAG-TTT-GTA-CAAAAA-AGC-AGG-CTA-CAT-GTG-GTC-TCA-TCC-TCA-ATTTGA-AAA-ATA-CCC-ATA-CGA-TGT-TCC-TGA-CTA-TG-3', and attB4-HA-R, 5'-GGG-GAC-AAC-TTT-GTA-TAG-AAA-AGTTGG-GTG-AGC-GTA-ATC-TGG-AAC-GTC-ATA-TGG-A-3'. The resulting polymerase chain reaction products were then gelpurified, using the QIAquick gel extraction kit (Qiagen), and the Invitrogen BP Clonase kit was then used to recombine the

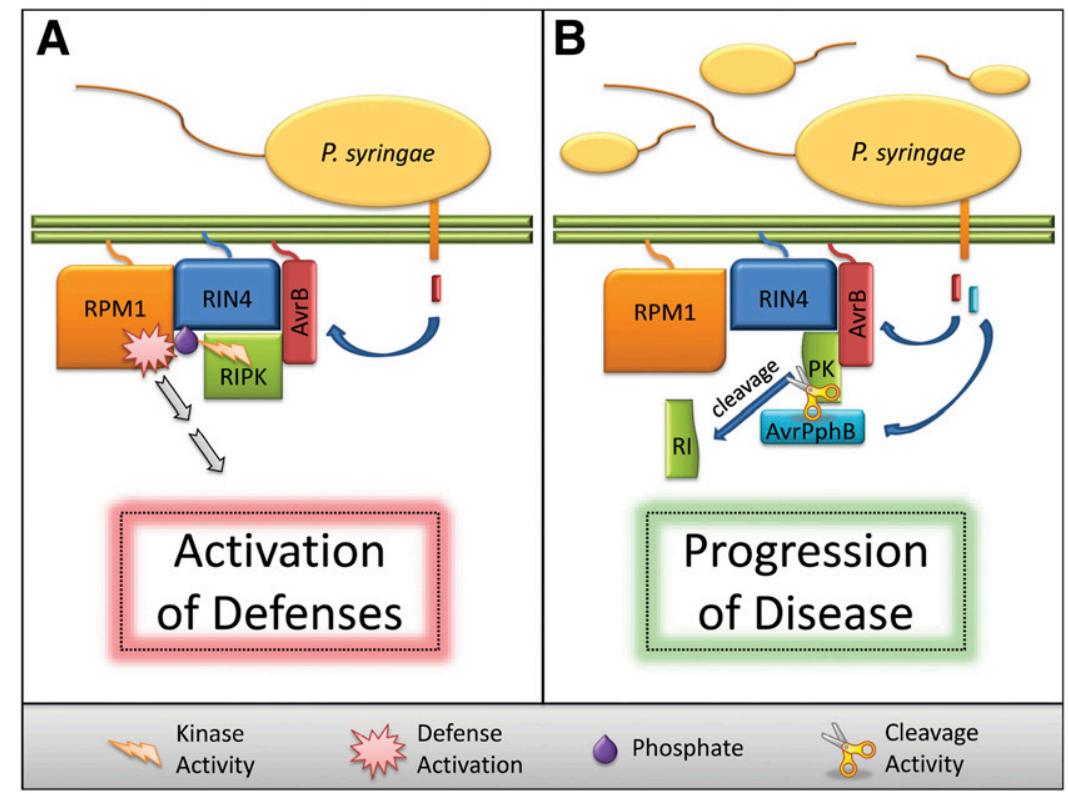

Fig. 9. Model for AvrPphB-mediated suppression of AvrB recognition. A, If AvrB is delivered into a host cell, it induces RPM1-interacting protein kinase (RIPK) to phosphorylate RIN4. RPM1 detects this modification and activates a hypersensitive response. B, If AvrPphB is delivered into the host cell along with AvrB, RIPK is cleaved by AvrPphB's protease activity. In the absence of RIPK, RIN4 does not get phosphorylated and RPM1 never becomes activated. Thus, bacteria that deliver both AvrB and AvrPphB have a growth advantage because AvrPphB can prevent AvrB from activating host defenses. 
products into the Gateway donor vector pBS-DONR(P1-P4) (Qi et al. 2012). The resulting clones were sequence-verified and the $R I P K$ construct was recombined with the strepII-HA tag into the Gateway-compatible expression vector pTA7002 (Aoyama and Chua 1997) using LR clonase (Invitrogen), following a multisite Gateway cloning procedure as previously described (Qi et al. 2012). All clones were sequenced to check for proper sequence and reading frame.

\section{Transient expression assays in $N$. benthamiana.}

For transient expression in $N$. benthamiana, Agrobacterium tumefaciens GV3101 (pMP90) strains transformed with the dexamethasone-inducible constructs described above were streaked onto Lura-Bertani (LB) plates containing $150 \mu \mathrm{g}$ of rifampicin per milliliter, $50 \mu \mathrm{g}$ of gentamicin per milliliter, and $100 \mu \mathrm{g}$ of kanamycin per milliliter. Single colonies were inoculated into $10 \mathrm{ml}$ of liquid LB gentamicin/kanamycin and were shaken overnight at $30^{\circ} \mathrm{C}$ and $250 \mathrm{rpm}$ on a New Brunswick rotary shaker. Cells were then subcultured 1:10 into fresh LB gentamicin/kanamycin and were shaken at $30^{\circ} \mathrm{C}$ for 5 to $6 \mathrm{~h}$, were spun down, and were resuspended in water to an optical density at $600 \mathrm{~nm}\left(\mathrm{OD}_{600}\right)$ of 1.0 (coexpression of multiple constructs was achieved by mixing strains in equal ratios). Bacterial suspension mixtures were infiltrated by needleless syringe into expanding leaves of 4- to 6-week-old $N$. benthamiana plants. Protein expression was induced by spraying the leaves with $50 \mu \mathrm{M}$ dexamethasone and $0.02 \%$ L77 surfactant (Momentive) $40 \mathrm{~h}$ after injection. Samples were collected for protein extraction every hour from 3 to $6 \mathrm{~h}$ after dexamethasone application and were flash-frozen in liquid nitrogen.

\section{Transient expression assays in $N$. glutinosa.}

For transient expression in $N$. glutinosa, we followed the protocol described by Kessens et al. (2014). In brief, A. tumefaciens GV3101 (pMP90) strains were grown overnight at $30^{\circ} \mathrm{C}$ in LB media with kanamycin and gentamycin $(50 \mu \mathrm{g} / \mathrm{ml}$ each). After overnight culture, bacterial cells were pelleted by centrifugation, were washed with $10 \mathrm{mM} \mathrm{MgCl} 2$, and were resuspended in $10 \mathrm{mM}$ $\mathrm{MgCl}_{2}$ containing $150 \mu \mathrm{M}$ acetosyringone and incubated at room temperature for at least $2 \mathrm{~h}$. Inoculum was prepared by mixing the indicated strains so that each transgene-containing strain had an individual $\mathrm{OD}_{600}=0.1$. The inoculum was injected into 3.5- to 4.5-week-old $N$. glutinosa plants with a needleless syringe, nicking the leaf surface with a razor blade prior to injection to facilitate fluid entry, when necessary. Older plants were avoided, as they transformed less reliably. Transgene expression was induced approximately $40 \mathrm{~h}$ postinjection by spraying the plants with $50 \mu \mathrm{M}$ dexamethasone and $0.02 \%$ L77 surfactant (Momentive). The leaves were scored for tissue collapse approximately $30 \mathrm{~h}$ later, and leaves were removed for photography shortly after. Expression of GmRIN4a and GmRIN4b was confirmed by immunoblot analysis.

\section{Immunoblot analysis.}

Frozen tissue was ground in liquid nitrogen and was resuspended in extraction buffer $(20 \mathrm{mM}$ Tris- $\mathrm{HCl}, \mathrm{pH} 7.5,150 \mathrm{mM}$ $\mathrm{NaCl}, 1 \mathrm{mM}$ EDTA, $1 \%$ Triton $\mathrm{X}-100,0.1 \%$ sodium dodecyl sulfate [SDS], $2 \mathrm{mM}$ 2,2-dithiodipyridine protease inhibitor [Sigma]). Total protein extracts were mixed with $4 \times$ SDS loading buffer at a ratio of 3:1 and were boiled for $5 \mathrm{~min}$ before separation by electrophoresis on a 4 to $20 \%$ gradient TrisHEPES-SDS polyacrylamide gel (Thermo Fisher Scientific). Proteins from duplicate gels were transferred to a nitrocellulose membrane at $80 \mathrm{~V}$ for $1 \mathrm{~h}$, were blocked in $5 \%$ dry milk in TBST (Tris-buffered saline with $0.1 \%$ Tween-20 [Sigma]) for $1 \mathrm{~h}$, and were probed with appropriate antibodies. For RIPK detection, membranes were incubated overnight in $5 \%$ dry milk buffer with 1:5,000 anti-HA:horseradish peroxidase (HRP) were washed three times for $10 \mathrm{~min}$ in TBST buffer, and were detected using the Immun-Star HRP substrate kit. For AvrPphB detection, membranes were incubated overnight in 5\% dry milk buffer with 1:100 anti-AvrPphB, were washed $3 \times$ three times for $10 \mathrm{~min}$ in TBST buffer, were incubated in 5\% dry milk buffer with 1:10,000 goat antirabbit HRP secondary antibody, and were detected using the Immun-Star HRP substrate kit.

\section{Bacterial growth curves.}

Analysis of $P$. syringae growth in Arabidopsis plants was performed on 5-week-old plants using syringe infiltration. Bacterial lawns of each strain were grown from single colonies selected on King's medium B (King et al. 1954) supplemented with $100 \mu \mathrm{g}$ of rifampicin per milliliter and $50 \mu \mathrm{g}$ of kanamycin per milliliter (for pVSP61 strains) or with $50 \mu \mathrm{g}$ of spectinomycin per milliliter (for pDSK600 strains). Bacteria were resuspended in $10 \mathrm{mM} \mathrm{MgCl} 2$ to a density of $\mathrm{OD}_{600}=0.0005$, and leaves were inoculated with each bacterial suspension by infiltration with a needleless syringe. Plants were returned to the growth room for $1 \mathrm{~h}$ before initial samples were taken. All data points shown in growth-curve plots represent the mean and standard deviation of three samples, with each sample consisting of four leaf disks $(0.5 \mathrm{~cm})$ from four separate leaves but ground in a single tube. The plants were returned to the growth room for four more days before a second time point was taken. Plant material was lysed using a Qiagen TissueLyser two times for $30 \mathrm{~s}$ at $30 \mathrm{~Hz}$. A 10-fold dilution series was generated from the lysate, and samples were plated at each dilution from $10^{\circ}$ to $10^{-5}$ on King's medium B with appropriate selection. Soybean growth curves were conducted in a similar manner as in Arabidopsis, except that plants were inoculated by dipping in a bacterial suspension at $\mathrm{OD}_{600}=0.5$ or by vacuum infiltration at $\mathrm{OD}_{600}=0.0005$. In either case, the bacterial suspension was supplemented with $0.025 \%$ Silwet L-77.

\section{ACKNOWLEDGMENTS}

We thank S.-H. Kim for providing primers for cloning and diagnostic testing. This work was supported by grants from the National Institute of General Medical Sciences of the National Institutes of Health (R01 GM046451) and National Science Foundation (IOS 1339348) and the Indiana University Faculty Research Support and Bridge Programs.

\section{LITERATURE CITED}

Ade, J., DeYoung, B. J., Golstein, C., and Innes, R. W. 2007. Indirect activation of a plant nucleotide binding site-leucine-rich repeat protein by a bacterial protease. Proc. Natl. Acad. Sci. USA 104:2531-2536.

Aoyama, T., and Chua, N.-H. 1997. A glucocorticoid-mediated transcriptional induction system in transgenic plants. Plant J. 11:605-612.

Ashfield, T., Keen, N. T., Buzzell, R. I., and Innes, R. W. 1995. Soybean resistance genes specific for different $P$ seudomonas syringae avirulence genes are allelic, or closely linked, at the RPG1 locus. Genetics 141:1597-1604.

Ashfield, T., Ong, L. E., Nobuta, K., Schneider, C. M., and Innes, R. W. 2004. Convergent evolution of disease resistance gene specificity in two flowering plant families. Plant Cell 16:309-318.

Ashfield, T., Redditt, T., Russell, A., Kessens, R., Rodibaugh, N., Galloway, L., Kang, Q., Podicheti, R., and Innes, R. W. 2014. Evolutionary relationship of disease resistance genes in soybean and Arabidopsis specific for the Pseudomonas syringae effectors AvrB and AvrRpm1. Plant Physiol. 166:235-251.

Axtell, M. J., and Staskawicz, B. J. 2003. Initiation of RPS2-specified disease resistance in Arabidopsis is coupled to the AvrRpt2-directed elimination of RIN4. Cell 112:369-377.

Axtell, M. J., Chisholm, S. T., Dahlbeck, D., and Staskawicz, B. J. 2003. Genetic and molecular evidence that the Pseudomonas syringae type III effector protein AvrRpt2 is a cysteine protease. Mol. Microbiol. 49:1537-1546.

Baltrus, D. A., Nishimura, M. T., Romanchuk, A., Chang, J. H., Mukhtar, M. S., Cherkis, K., Roach, J., Grant, S. R., Jones, C. D., and Dangl, J. L. 2011. Dynamic evolution of pathogenicity revealed by sequencing and 
comparative genomics of 19 Pseudomonas syringae isolates. PLoS Pathog. 7:e1002132.

Bisgrove, S. R., Simonich, M. T., Smith, N. M., Sattler, A., and Innes, R. W. 1994. A disease resistance gene in Arabidopsis with specificity for two different pathogen avirulence genes. Plant Cell 6:927-933.

Block, A., and Alfano, J. R. 2011. Plant targets for Pseudomonas syringae type III effectors: Virulence targets or guarded decoys? Curr. Opin. Microbiol. 14:39-46.

Charkowski, A. O., Huang, H. C., and Collmer, A. 1997. Altered localization of HrpZ in Pseudomonas syringae pv. syringae hrp mutants suggests that different components of the type III secretion pathway control protein translocation across the inner and outer membranes of gram-negative bacteria. J. Bacteriol. 179:3866-3874.

Chen, Z., Kloek, A. P., Cuzick, A., Moeder, W., Tang, D., Innes, R. W. Klessig, D. F., McDowell, J. M., and Kunkel, B. N. 2004. The Pseudomonas syringae type III effector AvrRpt2 functions downstream or independently of SA to promote virulence on Arabidopsis thaliana. Plant J. 37:494-504.

Chung, E. H., da Cunha, L., Wu, A. J., Gao, Z., Cherkis, K., Afzal, A. J., Mackey, D., and Dang1, J. L. 2011. Specific threonine phosphorylation of a host target by two unrelated type III effectors activates a host innate immune receptor in plants. Cell Host Microbe 9:125-136.

Deng, W. L., Preston, G., Collmer, A., Chang, C. J., and Huang, H. C. 1998 Characterization of the $h r p C$ and $h r p R S$ operons of Pseudomonas syringae pathovars syringae, tomato, and glycinea and analysis of the ability of $h r p F, h r p G, h r c C, h r p T$, and $h r p V$ mutants to elicit the hypersensitive response and disease in plants. J. Bacteriol. 180:4523-4531.

El Yacoubi, B., Brunings, A. M., Yuan, Q., Shankar, S., and Gabriel, D. W. 2007. In planta horizontal transfer of a major pathogenicity effector gene. Appl. Environ. Microbiol. 73:1612-1621.

He, P., Shan, L., Lin, N. C., Martin, G. B., Kemmerling, B., Nürnberger, T. and Sheen, J. 2006. Specific bacterial suppressors of MAMP signaling upstream of MAPKKK in Arabidopsis innate immunity. Cell 125:563-575.

Jones, J. D., and Dangl, J. L. 2006. The plant immune system. Nature 444: 323-329.

Kessens, R., Ashfield, T., Kim, S. H., and Innes, R. W. 2014. Determining the GmRIN4 requirements of the soybean disease resistance proteins Rpg1b and Rpg1r using a Nicotiana glutinosa-based agroinfiltration system. PLoS ONE 9:e108159.

King, E. O., Ward, M. K., and Raney, D. E. 1954. Two simple media for the demonstration of phycocyanin and fluorescin. J. Lab. Clin. Med. 44:301-307.

Liu, J., Elmore, J. M., Lin, Z. J., and Coaker, G. 2011. A receptor-like cytoplasmic kinase phosphorylates the host target RIN4, leading to the activation of a plant innate immune receptor. Cell Host Microbe 9:137-146.

Ma, W., Dong, F. F. T., Stavrinides, J., and Guttman, D. S. 2006. Type III effector diversification via both pathoadaptation and horizontal transfer in response to a coevolutionary arms race. PLoS Genet. 2:e209.

Mackey, D., Holt, B. F. I., 3rd, Wiig, A., and Dangl, J. L. 2002. RIN4 interacts with Pseudomonas syringae type III effector molecules and is required for RPM1-mediated resistance in Arabidopsis. Cell 108:743-754.

Mackey, D., Belkhadir, Y., Alonso, J. M., Ecker, J. R., and Dangl, J. L. 2003. Arabidopsis RIN4 is a target of the type III virulence effector AvrRpt2 and modulates RPS2-mediated resistance. Cell 112:379-389.

McCann, H. C., and Guttman, D. S. 2008. Evolution of the type III secretion system and its effectors in plant-microbe interactions. New Phytol. 177: 33-47.
Ong, L. E., and Innes, R. W. 2006. AvrB mutants lose both virulence and avirulence activities on soybean and Arabidopsis. Mol. Microbiol. 60: 951-962.

Qi, D., DeYoung, B. J., and Innes, R. W. 2012. Structure-function analysis of the coiled-coil and leucine-rich repeat domains of the RPS5 disease resistance protein. Plant Physiol. 158:1819-1832.

Qi, D., Dubiella, U., Kim, S. H., Sloss, D. I., Dowen, R. H., Dixon, J. E., and Innes, R. W. 2014. Recognition of the protein kinase AVRPPHB SUSCEPTIBLE1 by the disease resistance protein RESISTANCE TO PSEUDOMONAS SYRINGAE5 is dependent on s-acylation and an exposed loop in AVRPPHB SUSCEPTIBLE1. Plant Physiol. 164:340-351.

Roine, E., Wei, W., Yuan, J., Nurmiaho-Lassila, E. L., Kalkkinen, N., Romantschuk, M., and He, S. Y. 1997. Hrp pilus: An hrp-dependent bacterial surface appendage produced by Pseudomonas syringae pv. tomato DC3000. Proc. Natl. Acad. Sci. USA 94:3459-3464.

Rosebrock, T. R., Zeng, L., Brady, J. J., Abramovitch, R. B., Xiao, F., and Martin, G. B. 2007. A bacterial E3 ubiquitin ligase targets a host protein kinase to disrupt plant immunity. Nature 448:370-374.

Schechter, L. M., Roberts, K. A., Jamir, Y., Alfano, J. R., and Collmer, A. 2004. Pseudomonas syringae type III secretion system targeting signals and novel effectors studied with a Cya translocation reporter. J. Bacteriol. 186:543-555.

Selote, D., and Kachroo, A. 2010. RPG1-B-derived resistance to AvrBexpressing Pseudomonas syringae requires RIN4-like proteins in soybean. Plant Physiol. 153:1199-1211.

Selote, D., Robin, G. P., and Kachroo, A. 2013. GmRIN4 protein family members function nonredundantly in soybean race-specific resistance against Pseudomonas syringae. New Phytol. 197:1225-1235.

Shao, F., Golstein, C., Ade, J., Stoutemyer, M., Dixon, J. E., and Innes, R. W. 2003. Cleavage of Arabidopsis PBS1 by a bacterial type III effector. Science 301:1230-1233.

Simonich, M. T., and Innes, R. W. 1995. A disease resistance gene in Arabidopsis with specificity for the avrPph3 gene of Pseudomonas syringae pv. phaseolicola. Mol. Plant Microbe Interact. 8:637-640.

Swiderski, M. R., and Innes, R. W. 2001. The Arabidopsis PBS1 resistance gene encodes a member of a novel protein kinase subfamily. Plant J. 26 101-112.

Tsiamis, G., Mansfield, J. W., Hockenhull, R., Jackson, R. W., Sesma, A., Athanassopoulos, E., Bennett, M. A., Stevens, C., Vivian, A., Taylor J. D., and Murillo, J. 2000. Cultivar-specific avirulence and virulence functions assigned to avrPphF in Pseudomonas syringae pv. phaseolicola, the cause of bean halo-blight disease. EMBO (Eur. Mol. Biol. Organ.) J. 19:3204-3214.

Zhang, J., and Zhou, J. M. 2010. Plant immunity triggered by microbial molecular signatures. Mol. Plant 3:783-793.

Zhang, J., Li, W., Xiang, T., Liu, Z., Laluk, K., Ding, X., Zou, Y., Gao, M., Zhang, X., Chen, S., Mengiste, T., Zhang, Y., and Zhou, J. M. 2010. Receptor-like cytoplasmic kinases integrate signaling from multiple plant immune receptors and are targeted by a Pseudomonas syringae effector. Cell Host Microbe 7:290-301.

\section{AUTHOR-RECOMMENDED INETERNET RESOURCE}

United States Department of Agriculture soybean germplasm collection: sun.ars-grin.gov/npgs 